\title{
Rejoua aurantiaca. Bernard Juillerat et la botanique
}

\section{Christian Coiffier}

\section{(2) OpenEdition}

\section{Journals}

\section{Édition électronique}

URL : http://journals.openedition.org/jso/6108

DOI : 10.4000/jso.6108

ISSN : $1760-7256$

\section{Éditeur}

Société des océanistes

\section{Édition imprimée}

Date de publication : 15 décembre 2010

Pagination : 161-172

ISBN : 978-2-85430-027-7

ISSN : 0300-953x

Référence électronique

Christian Coiffier, «Rejoua aurantiaca. Bernard Juillerat et la botanique », Journal de la Société des Océanistes [En ligne], 130-131 | 2010, mis en ligne le 15 décembre 2013, consulté le 12 juin 2020. URL: http://journals.openedition.org/jso/6108; DOI : https://doi.org/10.4000/jso.6108 


\title{
Rejoua aurantiaca. Bernard Juillerat et la botanique
}

\author{
par
}

\section{Christian COIFFIER*}

\section{RÉSUMÉ}

Les sociétés du Sépik en Papouasie Nouvelle-Guinée utilisent pour leurs rituels des fruits qui ont la particularité de changer de couleur durant leur maturation. Ils ont été souvent perçus comme de simples décorations. Bernard Juillerat a essayé de comprendre ce qu'ils représentaient vraiment pour la société yafar du Sépik occidental. Nous comparerons ses données aux nôtres que nous avons recueillies chez les Iatmul et les Sawos du Sépik oriental.

MotS-CLÉS: ethnobotanique, Sépik, maturation, fruits orange, Rejoua, Ervatamia

Tout chercheur en sciences sociales qui étudie une société rurale se doit d'avoir des connaissances sur le monde végétal. On peut regretter que nombre d'ethnologues n'aient pas jugé utile de réaliser des identifications scientifiques des végétaux (ou des animaux) qu'ils évoquent dans leurs écrits. Ce qui ne permet pas d'utiliser leurs travaux pour des recherches comparatives, l'essentiel étant de s'appuyer sur des connaissances botaniques fiables pour comparer les mêmes espèces végétales. Le rapprochement des usages de certaines plantes dans un ensemble de sociétés voisines, situées dans des zones écologiques similaires, permet en effet de décrypter les différences existant dans les diverses façons qu'ont ces sociétés d'organiser leur vision du monde.

Bernard Juillerat avait bien compris cela et il trouva auprès de Jacques Barrau, du Muséum national d'histoire naturelle, l'aide nécessaire pour acquérir les connaissances botaniques qui

\section{ABSTRACT}

For their rituals Sepik societies in New-Guinea use fruits which characteristically change color during their maturation. These fruits were often conceived as mere decorations. Bernard Juillerat tried to unterstand what they really represented for the Yafar society of Western Sepik. We will compare his data with ours which we collected with the Iatmul and Sawos Societies of East Sepik.

KEYwORDs: ethnobotany, Sepik, ripening, orange fruit, Rejoua, Ervatamia

lui manquaient. Sa bibliographie ( $c f$. Leblic dans ce volume) montre l'intérêt qu'il porta aux végétaux de Papouasie Nouvelle-Guinée à une certaine époque de sa carrière lorsqu'il publia successivement, de 1982 à 1984, quatre articles dans le Journal d'agriculture traditionnelle et de botanique appliquée avant de réaliser, en 1986, son fameux ouvrage Les enfants du sang: société, reproduction et imaginaire en Nouvelle-Guinée. J'eus l'occasion de lire cet ouvrage peu avant de partir «faire mon terrain » en 1987-1988, chez les Iatmul et les Sawos du Sépik-Est en Papouasie Nouvelle-Guinée, deux sociétés qui vivent dans une région relativement proche de celle des Yafar. Le présent article me donne l'occasion de m'acquitter d'une dette envers Bernard Juillerat qui m'a ainsi encouragé à focaliser mes recherches sur certains végétaux qu'il avait lui-même étudiés dans un contexte différent. Je fus donc à même de poser à mes informateurs des questions

* Maître de conférences au MNHN, coiffier@mnhn.fr 
plus pertinentes au sujet de diverses plantes productrices de fruits orange (Rejoua aurantiaca) utilisés dans de nombreux rituels. C'est ainsi que j'ai compris que leur valeur "symbolique » dépassait de beaucoup l'aspect esthétique que de nombreux observateurs s'accordaient à leur reconnaitre. La littérature ethnographique sur le Sépik abonde d'histoires relatives à ces fruits ; malheureusement, peu d'auteurs ont fourni des précisions sur leurs représentations locales. À ma connaissance, Bernard Juillerat est le seul à avoir essayé de comprendre de façon rigoureuse la perception profonde de ces fruits orange dans la mentalité de ses informateurs yafar.

Malgré tout, il faut être prudent dans les comparaisons car l'observation d'un usage similaire

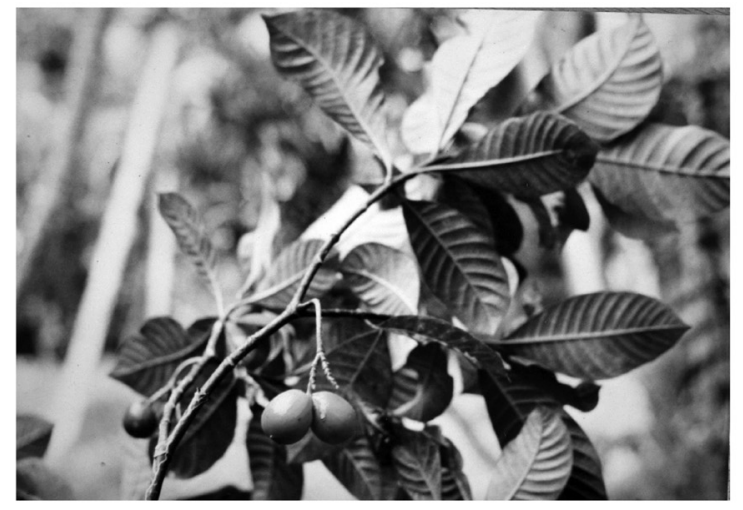

Рното 1 - Rameau de Rejoua aurantiaca (cliché de l'auteur)

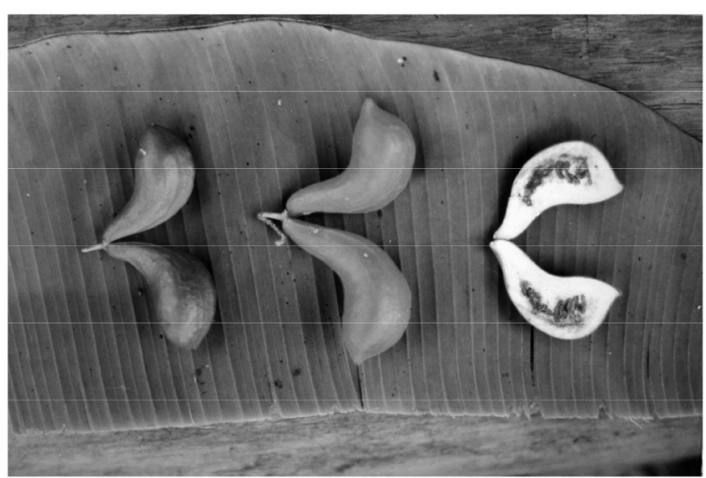

Рното 3. - Détails des fruits jumeaux de Rejoua aurantiaca : a) fruits vert ; b) fruits orange matures ; c) section longitudinale (cliché de l'auteur)

\section{Description botanique des plantes rituelles à fruits orange (région du Sépik)}

Le Rejoua aurantiaca Gaud., famille des Apocynaceae, a été décrit pour la première fois par Freycinet, en 1826. Le genre Rejoua est nommé Tabernaemontana par certains botanistes. Selon ces derniers, il en existerait d'une à trois espèces décrites en Papouasie Nouvelle-Guinée (van Royen, 1969 : 43-45). L'aire d'expansion de ce dans deux sociétés voisines n'implique pas forcément une même perception de l'objet, ici les fruits de l'arbuste Rejoua aurantiaca. Après avoir présenté les divers genres de fruits orange utilisés par les populations du Sépik, je m'attacherai à rassembler, dans l'œuvre de Bernard Juillerat, les données éparses relatives à la perception de ces fruits par ses informateurs. J'essaierai ensuite d'exposer leurs représentations chez les Iatmul et les Sawos du Sépik oriental avant d'élargir mon enquête aux sociétés voisines. Je conclurai cet hommage à Bernard Juillerat en rassemblant diverses représentations de ces plantes communes à l'ensemble des populations des deux provinces du Sépik.

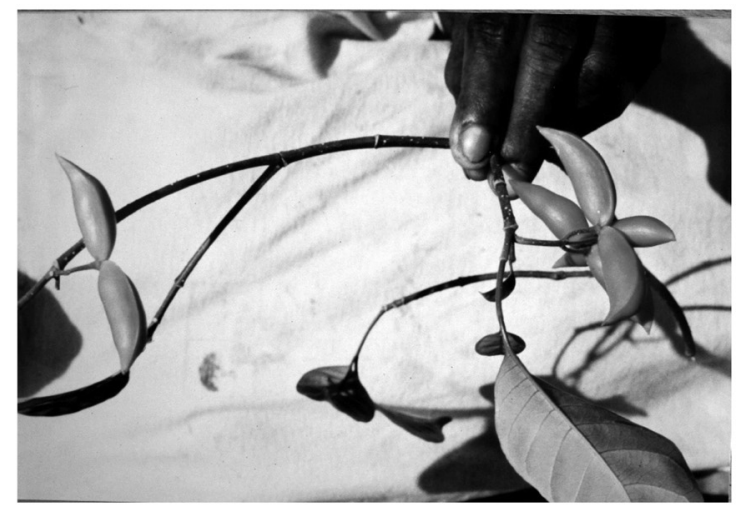

Рното 2. - Rameau d'Ervatamia orientalis (cliché de l'auteur)

genre s'étend de l'île de Ceram (à l'ouest) jusqu'au nord du Vanuatu (à l'est), en englobant une partie de l'île de Nouvelle-Guinée. Il est également présent aux îles Salomon. Les arbustes de Rejoua aurantiaca sont de taille moyenne. Ils donnent des fleurs blanches et produisent en abondance, en janvier et septembre, des fruits indéhiscents qui se détachent de leurs branches à maturité pour tomber sur le sol. Ils sont constitués, une fois mûrs, d'une enveloppe à paroi externe rigide et lisse de couleur orangée vive avec une paroi interne molle et duveteuse blanche. Les fruits de Rejoua ont la particularité de passer par plusieurs couleurs au cours de leur développement : ils sont d'abord blanc-vert, puis virent au jaune pour devenir orange vif (voire rouge) ; une fois tombés au sol, ils deviennent noirs en pourrissant. Ces fruits contiennent en moyenne de dix à vingt petites graines noires ou brunes, mais peu de pulpe. Se présentant toujours par paires à l'extrémité des branches, ils ne sont pas comestibles (Cooper, 2004 : 54-55). Je montrerai ultérieurement que cette particularité de regroupement par paires est cruciale pour des peuples qui accordent une grande importance à la dualité. Les chimistes ont trouvé dans l'écorce 
du Rejoua aurantiaca plusieurs alcaloïdes : iboluteïne, vobtusine, voaluteïne et voacangine. On sait que la voacangine extraite d'une Apocynaceae africaine a permis par hémisynthèse ${ }^{1} \mathrm{~d}^{\text {'être }}$ utilisée positivement dans le traitement de certains types de leucémie (Guise et al., 1965 : 92731). Le Rejoua aurantiaca a des propriétés antibactériennes et particulièrement antivirales contre l'Herpes simplex. Dans la région du Sépik, sa sève était appliquée sur les ulcères tropicaux ou les mauvaises blessures pour en extraire le «mauvais sang ».

Un autre petit arbuste de sous-bois de moins de deux mètres de hauteur et croissant sur les terrains calcaires, l'Ervatamia, appartient également à la famille des Apocynaceae et produit des fruits orange utilisés pour certains rituels. L'aire de diffusion de ce végétal est beaucoup plus vaste que celle du Rejoua. Elle s'étend de l'Asie du Sud-Est jusqu'à la Polynésie. Les Ervatamia sont particulièrement abondants aux Philippines, en Indonésie de l'Est, en Nouvelle-Guinée et sur la côte nord-est de l'Australie. Il en existe quatrevingt-dix espèces dans le monde dont au moins trois en Papouasie Nouvelle-Guinée : Ervatamia eriophora, Ervatamia pubescens, Ervatamia orientalis. Il faut ajouter l'espèce Ervatamia coronaria qui est cultivée pour ses qualités ornementales. Le fruit de l'Ervatamia sp. n'est pas comestible et présente deux méricarpes de couleur orangée ou jaune ressemblant à deux petites cornes opposées (photo 2) (van Royen, 1969 : 24-27; Allorge, 1985). Les fruits déhiscents s'ouvrent sur la zone de suture des carpelles et laissent apparaître six à huit graines brunes entourées d'une pulpe rouge. Alors que les graines de Rejoua tombent sur le sol, celles de l'Ervatamia sont disséminées soit par les fourmis, soit par les oiseaux. Les tiges brisées de tous ces arbustes laissent suinter un latex blanchâtre. Ces végétaux donnent des fleurs blanches en janvier et septembre, puis fructifient en mars et novembre. Dans le contexte du présent article, un autre arbuste présente un intérêt pour la forme et la couleur de ses fruits, le Voacanda papuana. Il en existe deux espèces en Nouvelle-Guinée (Cooper, 2004 : 56) dont les fruits n'acquièrent jamais l'intensité de la couleur orange des fruits de Rejoua aurantiaca (van Royen, 1969: 28-30, 47-48).

Il est étonnant de ne pas trouver trace de ces végétaux éminemment rituels dans le chapitre «Ethnobotany », écrit par J. M. Powell, dans l'ouvrage New Guinea Vegetation (Paijmans, 1976). L'explication vraisemblable est le manque relatif, dans cet ouvrage, de documentation sur les régions des Basses Terres par rapport à la masse des références concernant les Hautes Terres. Dans le monde asiatique bouddhiste confucianiste, les fleurs blanches d'Ervatamia sont utilisées en association avec les fleurs de frangipanier (Plumaria sp.) comme offrandes sur les autels. Le frangipanier appartient également à la famille des Apocynaceae. Ces fleurs représentent un symbole de pureté qui accompagne les offrandes alimentaires aux ancêtres.

\section{Le fruit de Rejoua aurantiaca chez les Yafar du Sépik occidental}

Dans les représentations de nombreux peuples de Mélanésie, le processus de mûrissement de ces fruits se trouve perçu en relation analogique avec les diverses phases de la vie humaine: de la naissance à l'adolescence et de la maturité à la mort. Tous les peuples du Sépik admirent ces fruits et les utilisent dans leurs rituels. Les références à l'arbre Rejoua aurantiaca apparaissent dans plusieurs ouvrages de Bernard Juillerat. Celui-ci a très bien su trouver la place occupée par les fruits de ce végétal dans l'univers mental des Yafar du Sépik-Ouest. Selon lui, les différentes couleurs associées aux diverses phases du mûrissement des fruits de Rejoua sont perçues comme une permanente " métaphorisation » de l'homme par le végétal. Celle-ci représenterait les transformations de la personnification du « moi » (sungwaag) au cours d'une vie humaine. Les Yafar avaient une notion très importante, le hoofuk, qu'ils définissaient d'une part comme l'intérieur de la matière molle et putrescible comme la moelle des palmiers ou la chair des ignames et, d'autre part, comme un principe de fécondité (Juillerat, 1986 : 241, 547). L'idée de mûrissement ou de maturation, qui est associée à celle de caducité, est exprimée par le terme abuk qui a deux sens : la couleur rouge et le fait d'être mûr. Être mûr, c'est être proche de la chute et du pourrissement donc de la mort (Juillerat, 1986 : 241, 364, 547). Il devient vulnérable et suscite des agressions diverses. Aussi les Yafar disent que le sungwaag d'une personne est boof abuk c'est-àdire " mûr" comme un fruit boof. Les Yafar opposent à l'arbre boof (Rejoua aurantiaca) l'arbuste gungwan (Antiaropsis sp.) dont les fruits ne tombent pas et finissent par pourrir sur pied (Juillerat, $1991: 215$ ).

1. NDLR. - En chimie, une hémisynthèse est la synthèse d'une molécule réalisée à partir de composés naturels possédant déjà une partie de la molécule visée. 
«L'état intermédiaire entre la fermeté du fruit vert ou du moi "noir" et leur maturité complète est désigné par le terme heweheg "à demi-mûr". " (Juillerat, 1986 : 364)

De même, on dira que le sungwaag d'une personne contaminée par une maladie est heweheg. $\mathrm{Si}$ aucun rite ne peut arriver à améliorer sa situation, elle se dégradera peu à peu vers un état de boof abuk et évoluera vers la mort.

« Il en va de même pour le gibier que l'on rend magiquement abuk afin qu'il tombe sous les traits du chasseur. » (Juillerat, 1986 : 364)

Pour les Yafar, la vision de la mort est duelle :

«Le monde se scinde en deux types d'entités : la première est le fantôme (ifaaf) qui n'est que le double ou le moi (sungwaag) de la personne transformée par la mort, la seconde est l'esprit du mort proprement dit (nabasa), qui se présente sous deux modes, l'esprit du sang ou l'esprit des os du défunt. » (Juillerat, 1991 : 200)

"L'autre mode d'identification mortifère est d'ordre spatial : le sungwaag non seulement va "voir les nabasa", mais tend parfois à descendre dans le monde plus profond des morts qui est aussi celui, maternel, de la fertilité première, du hoofuk ou du "sang" primordiaux. L'association cosmologique de la mort et du pourri (ifaaf) avec le hoofuk originel prend un sens supplémentaire dans la médiation du sungwaag qui, contaminé, rapporte à son propriétaire une double promesse de mort et de renaissance. » (Juillerat, $1986: 363-364$ )

Les fruits boof représentent donc pour les Yafar une image de la régénération des êtres et des végétaux.

Lors des rites funéraires, le crâne et certains ossements du défunt étaient placés dans la fourche d'un arbre, habituellement un Gnetum gnemon (tulip en pidgin, masiy en amanab) qui, en croissant, élevait ces reliques dans l'épaisseur de la végétation forestière (Juillerat, 1986: 401). Cet arbuste se trouve en général associé en Papouasie Nouvelle-Guinée à la dualité à cause de la disposition opposée de ses feuilles. Lorsque le défunt descend dans le monde souterrain par une longue route, il arrive devant une bifurcation à peine visible avec un chemin qui remonte et l'autre qui descend à l'intérieur de la terre. S'il choisit le second, il rencontrera à l'entrée du monde des morts un arbre boof (Rejoua aurantiaca) dont les fruits représentent la maturité et la caducité, préalables au pourrissement, mais prélude à la régénération. Auprès de cet arbre se trouve le père terrible du monde souterrain, l'esprit de la mort, qui d'un coup de bâton brisera son nez en le transformant ainsi en spectre (Juillerat, 1991 : 201； 1992 : 115).

« Le mûrissement du moi est ici l'effet immédiat recherché dans la sorcellerie non létale; le principe identificatoire avec l'arbre aux fruits caduques entraîne la chute vers le monde souterrain, c'est-à-dire le retour incestueux à la mère génitrice. » (Juillerat, 1991: 210)

« La Mère mauvaise et fertile est d'ailleurs gardée par le "mauvais père" Wangohra qui a pour signe l'arbre boof aux fruits caducs. » (Juillerat, 1986 : 404)

Un mythe yafar indique que l'arbre boof aux fruits rouges aurait pour origine le sang menstruel de la Grand-mère originelle (Juillerat, 1992: 115). Dans les cas de meurtre de contresorcellerie, un rite avait lieu auprès de l'abri funéraire qui était décoré pour la circonstance de pieux peints en rouge, de fruits boof associés à des feuilles rouges de croton et à du jus de bétel (Juillerat, 1986: 401).

Les Yafar utilisent les fruits orange boof comme parures lors des cérémonies yangis qui annoncent l'imminente naissance des " enfants du sang »(Juillerat, 1986 : 77, 364). Ils en placent des guirlandes autour des masques ageli surmontés de feuilles de sagoutier ou s'en servent de décoration corporelle (Juillerat, 1986: 393; 1992: 28-29, 35).

«Les Yafar disent que les ornements rendaient la chasse plus fructueuse [...] bien que la beauté, les peaux propres et frottées de graisse de porc [...] soient déjà la condition préalable de l'accès à l'abondance. » (Juillerat, $1986: 393$ )

Les masques amof ou termites, avec la tête emballée dans une écorce jaunie au Curcuma, portent des parures de feuillages et de fruits boof qui évoquent par leurs teintes le mûrissement et la caducité. Cette interprétation est confirmée par un mythe décrivant le héros culturel Wefroog faisant tomber les fruits boof de leur arbre et s'en servant pour confectionner le personnage amof auquel il ordonne d'aller danser pour la cérémonie yangis (Juillerat, 1995: 61；1992 : 51). Contrairement aux autres personnages masqués, les masques termites dansent avec les femmes et les enfants. Selon l'exégèse des experts yafar, ils viennent annoncer l'imminente naissance des fils totémiques, " mûrs " comme des fruits prêts à se détacher. La grosse tête ronde des hommes masqués évoque le termite, mais aussi la tête d'un foetus (Juillerat, 1995 : 61). Les fruits orange de Rejoua semblent donc perçus comme des marqueurs de l'évolution biologique des hommes. La relation avec le fœetus est à rapprocher de celle du masque mbamba des Abelam qui est associé au placenta et qui se présente toujours 
avec un collier de fruits orange de Rejoua. De même chez les Iatmul, durant un rituel naven pour un homme adopté par un clan différent du sien, un membre de sa famille biologique travesti en femme danse avec un collier de fruits orange (Coiffier, 1994 : 246-247). Ce collier est la marque de son attachement à ses ancêtres biologiques.

Les recherches d'Alfred Gell, qui a travaillé dans la société des Umeda voisine des Yafar, viennent préciser le sens des représentations de ce fruit orange dans la région. Gell fait remarquer (Gell, 1975: 314-315) l'homonymie entre subove, désignant le fruit de Rejoua en langue umeda, avec l'adjectif subove utilisé dans la formation des termes de couleur : vert, rouge, jaune, pourpre. De plus, le champ sémantique de la racine $s u b$ - est révélateur du sens de la représentation de ce fruit dans cette société : sub désigne le palmier limbum; subul, un ver de terre ; subudagwa, un esprit souterrain de la croissance et subof, le pigeon Goura (Goura victoria). L'étude botanique de différents végétaux utilisés pour les masques des rites de fertilité permet de comprendre ces associations sémantiques. Le palmier limbum est un élément central lié à l'esprit souterrain, subudagwa, responsable de la croissance des plantes associé aussi par analogie au ver de terre subul qui ne cesse de remuer le sol. La position des guirlandes de fruits orange sur divers types de masques correspond à la place habituelle des fruits de palmier (cocotier, aréquier, limbum) au sommet du stipe et sous les palmes (Gell, 1975 : 241). Quant à la relation avec le pigeon Goura, il semble d'après Gell qu'il faille la trouver dans une analogie avec la couleur pourpre des yeux de cet oiseau.

Les informations collectées par Gell chez les Umeda confirment donc la relation de ces fruits orange avec l'esprit souterrain responsable de la croissance et de la régénération évoquée par Juillerat chez les Yafar (Juillerat, 1986: 404 ; 1991 : $201 ; 1992$ : 115).

\section{Les fruits orange chez les Iatmul et les Sawos du Sépik oriental}

Les Iatmul et les Sawos classent dans la catégorie des mbwandi différents genres et espèces de végétaux à fruits orange qui présentent des formes très diverses dont plusieurs sortes de Rejoua des Sawos. Littéralement, le mot mbwandi serait constitué du mot mbwan qui désigne un esprit ancestral représenté sous la forme d'une pierre dressée et de $n d i$, excréments. Ces pierres sont dressées sur des monticules (waak) situés devant les pignons des grandes maisons cérémonielles. C'était sous ces monticules qu'étaient enterrés jadis les restes des ennemis tués (Coiffier, 1994 : 1443-1467). La prospérité d'un village était censée dépendre de ces mbwan. Selon Bateson, le mot mbwan désignerait également, dans certains cas, l'esprit de l'ennemi tué qui était parfois considéré comme un ancêtre parce qu'il contribuait à la prolifération de la communauté. Dans le langage des chamans, l'expression pour la copulation est mbwan-tou «dresser une pierre » (Bateson, 1971 : 151, 291).

Les diverses variétés de fruits mbwandi sont distinguées par un terme spécifique selon leur ressemblance analogique avec les noix de coco, des animaux aquatiques : canard, anguille, serpent, poisson-chat, ou des éléments d'animaux : défense de porc, œuf de tortue. Les fruits non mûrs de couleur verte sont appelés djepma mbwandi, ceux de couleur orange aak mbwandi (ou kuu mbwandi) chez les Sawos et ceux de couleur noire nguel mbwandi.

TABLEAU 1. - Les diverses variétés de mbwandi chez les Iatmul et les Sawos

\begin{tabular}{|c|c|}
\hline tepma mbwandi & de tepma, cocotier, Rejoua aurantiaca; gros fruit rond de six centimètres de diamètre \\
\hline mboïkala mbwandi & 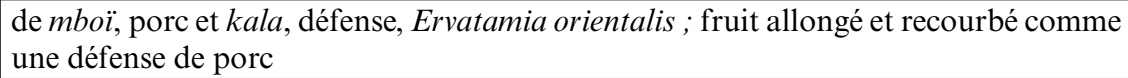 \\
\hline nguse & $\begin{array}{l}\text { de } n g u s e, \text { tortue et mbande, œuf, Rejoua aurantiaca; petit fruit rond comme un œuf de } \\
\text { tortue de trois centimètres de diamètre }\end{array}$ \\
\hline dewatupmui mbwandi & de dewa, canard et tupmui, bec, Rejoua sp. ; fruit recourbé comme un bec de canard \\
\hline ango mbwandi & de ango, petite anguille, Rejoua novoguineensis; fruit en tortillon \\
\hline kambaï mbwandi & de kambaï, serpent, Rejoua novoguineensis ; fruit allongé comme u1 \\
\hline kami mbwandi & on-chat, Rejoua longipedunculata; tout petit fruit \\
\hline wué mbwandi & Voacanga papuana; avec des gros fruits en forme de figue \\
\hline kulagwa mbwandi* & $\begin{array}{l}\text { Capsicum frutescens; petit piment rouge classé dans la catégorie des mbwandi par les } \\
\text { Iatmul et les Sawos }\end{array}$ \\
\hline
\end{tabular}

* Excepté le Capsicum frutescens, tous ces végétaux appartiennent à la famille des Apocynaceae. 
Une espèce de palmier (Orania sp.) considérée par les Iatmul comme un cocotier sauvage est appelée mbwandirepma tepma car ses fruits ressemblent à ceux du mbwandi (Rejoua aurantiaca). Les arbustes tepma mbwandi et mboïkala mbwandi ont environ cinq mètres de hauteur ; il en existe des formes sauvages et d'autres cultivées (Ervatamia coronaria). Les arbustes mbwandi sont plantés généralement sur les tertres qui entourent la place d'un village et parfois sur les waak devant les pignons des maisons cérémonielles.

Chacun des clans, chez les Iatmul comme chez les Sawos, peut utiliser certaines variétés de ces fruits qui sont considérés comme des substituts des ennemis assassinés et parfois d'ancêtres. Ces fruits sont choisis en fonction de leur forme pour de multiples usages. Jadis, à l'époque des guerres intercommunautaires, la tête coupée d'un ennemi était empalée sur un bambou et plantée sur le monticule waak à côté des mbwan et entourée d'une petite clôture agrémentée de fruits $m b w a n d i$. La tête était alors censée danser. Cette pratique se retrouvait dans la région de Maprik où des fruits de ban (Rejoua sp.) orange étaient empalés avec un crâne sur un bambou placé auprès des pierres rituelles de la maison des hommes (Aufenanger, 1972: 283). Il existe manifestement une relation analogique entre la caducité de ces fruits et la décapitation. Ces fruits, de toutes variétés, étaient utilisés en grand nombre durant les cérémonies des secondes funérailles appelées mindjangu. Les côtés de la plate-forme supportant le mannequin avec le crâne surmodelé du défunt étaient " décorés » de guirlandes festonnées réalisées avec des fruits ovoïdes mbwandi verts, jaunes et rouges enfilés sur une éclisse de rotin. Des fruits mbwandi étaient également enfilés sur de longues baguettes alors que d'autres en forme de tortillons ango mbwandi et kambaï mbwandi étaient placés aux extrémités d'un faisceau de bâtonnets au-dessus du crâne surmodelé. Le plastron placé sur la poitrine du mannequin était aussi décoré de la sorte avec des fruits d'Ervatamia et de Rejoua suivant une artistique composition. Le visage reconstitué du défunt était agrémenté de fruits ronds de Rejoua aux deux extrémités de sa parure nasale en dents de porc sauvage recourbées. Durant les autres rituels ces fruits se trouvent fréquemment empalés à l'extrémité de la pointe d'une lance ou d'un flambeau tuaï. Les Sawos s'en servent également pour "décorer» les panneaux mbowi sakué peints sur des palmes de sagoutier. Chacun de ces fruits orange représente un ennemi tué pour la prospérité de la communauté par un membre du clan proprié- taire de l'objet ou de la cérémonie. Certains prétendent que ces fruits seraient des représentations des crânes de ces ennemis car la partie interne de leur enveloppe ressemble selon eux à la partie interne d'une boîte crânienne. Cette vision des choses est associée dans la pensée iatmul à l'idée de beauté. Il est indéniable que les couleurs lisses et brillantes de ces fruits présentent une connotation esthétique qui participe à donner une impression festive destinée à plaire tant aux esprits des eaux wagan qu'aux esprits sylvestres wundjumbu équivalents des esprits nabasa des Yafar.

Jadis, lors des grandes cérémonies wagan mbangu, deux grands mannequins recouverts de végétaux divers, dont de nombreux fruits orange mbwandi, personnifiaient les esprits des eaux wagan (Bateson, 1971 : planche 17). Les cérémonies d'inauguration des maisons familiales ou cérémonielles sont encore de nos jours l'occasion d'utiliser ces fruits orange. Ils sont alors enfilés par paires symétriques sur les nervures d'une feuille de sagoutier dont le rachis est placé dans la bouche du masque de pignon. Cette « décoration » est appelée samambwandi chez les Sawos de Torembi et de Marap et ngeko mbwandi chez les Iatmul de Kandingaï. Elle demeure en place après la fête jusqu'au moment où les fruits perdent progressivement leur belle couleur pour devenir noirs et desséchés. De nombreux poteaux de maison présentent des couronnes de mbwandi sculptés dans le bois à différents niveaux. Ces fruits sont présents lors de toutes les cérémonies importantes. On les retrouve ainsi associés aux sculptures de pignons représentant des ancêtres et sur divers types de masques dont les mwaï. Les tabourets d'orateur des maisons cérémonielles sont également agrémentés avec ces fruits pour les jours de fête. En fait, chaque clan les utilise de façon différente. Lors de naven importants, un oncle maternel wau travesti en femme peut se présenter avec une guirlande (mogul mbwandi) de fruits orange ovoïdes (Rejoua aurantiaca) associée à une houppe de plumes accrochée dans l'entre-jambes. Lorsque ce dernier danse, il met celle-ci bien en évidence (Coiffier, 1994 : 793, 1369). Cette pratique est cependant réservée aux membres de certains clans. Gregory Bateson (1971 : 27) décrit une scène similaire durant laquelle un wau se placerait dans l'anus un fruit de mbwandi représentant, dans ce cas, un " clitoris anal ». Aucun de mes informateurs ne m'a confirmé cette histoire, mais il est possible que Bateson, qui n'avait pas vu cette scène, l'ait interprétée à sa manière.

Les fruits de Rejoua sont fréquemment utilisés pour la divination. Dans un mythe recueilli à 
Tambanum, un homme plonge pour aller se rendre compte de l'état du crocodile qu'il vient de transpercer. Il dit à ses compagnons de pirogue qu'il laissera remonter à la surface de l'eau un fruit orange si l'animal est mort ou un fruit vert si ce dernier n'est que blessé2. Aufenanger (1972: 357) relate une histoire similaire recueillie dans la région de Kaugia. Le clan Suaru possède une magie pour la pêche. Des feuilles de gingembre et de curcuma sont enveloppées dans une spathe de palmier avec des fruits de Rejoua enfilés sur les ligatures pour constituer un paquet magique qui est jeté dans l'eau d'une rivière pour attirer les poissons et les chevrettes dans les chenaux et les étangs (Coiffier, 1994 : 794-795). La variété ango mbwandi (litt. anguille-Rejoua) est utilisée pour pêcher les petites anguilles. Comme pour les autres espèces, les fruits de Rejoua novoguineensis sont groupés par paires à l'extrémité des rameaux, un fait qui est à mettre en relation avec un mythe du clan Suaru qui évoque un couple d'anguilles constitué de deux frères nommés Malikaman et Suatkaman qui seraient à l'origine du fleuve Sépik. Ces fruits flottent très facilement, d'où l'utilisation actuelle du mot mbwandi pour nommer les flotteurs en plastiques ou en liège des nouveaux filets de pêche introduits dans la région durant les années 1960.

Le latex, très abondant, produit par les tiges de Rejoua aurantiaca est utilisé comme colle et à des fins médicales. Le clan Suaru possède une technique particulière pour fabriquer un poison à partir de ce latex. Les gens de langue Sanio en amont du fleuve utilisent également ce latex pour empoisonner les poissons. Autrefois la sève de mbwandi était utilisée pour réaliser les tatouages des femmes et elle était considérée comme excellente pour soigner les ampoules des pieds. Elle pouvait également être diluée dans de l'eau chaude pour être bue comme remède à certains maux de ventre. La dose de sève diluée dépendait alors de l'âge du malade. Ces divers exemples montrent que chaque genre ou espèce de fruits orange utilisés correspond à un usage particulier qui, parfois, demeure la propriété de certains clans.

\section{Perception des fruits orange, ailleurs, dans la région du Sépik}

Les Abelam, qui vivent sur des collines situées entre la mer et le fleuve Sépik, font un grand usage rituel des fruits orange mban (Rejoua aurantiaca) qui ont diverses représentations. Selon Forge (1971 : 305), ils sont les symboles des ennemis morts et sont associés lors de certaines danses à des « décorations » faites de morceaux d'efflorescences de palmier blanc et de feuilles rouges symbolisant les conquêtes sexuelles de femmes étrangères par la communauté. Dans d'autres cas, ils servent à compter les victimes revendiquées par un village (Forge, 1971 : 304). Ils peuvent également représenter le nombre de porcs sacrifiés durant une cérémonie (Hauser-Schäublin, communication personnelle). Lors des danses des masques mbamba, les fruits mban en guirlandes autour de leur cou, sont associés à d'autres végétaux selon les clans : feuilles de taro, feuilles de cordyline pliées en accordéon, frondes de fougère, gaine blanche de feuilles de Crinum asiaticum ou hibiscus (Gardi, 1958 : 141, 143 ; Kirk, 1973 : 370-371). Dans les coiffures, les frondes de sélaginelles qui leur sont associées sont signes d'homicide (HauserSchäublin, 1980 : 17.2 ; Losche, 1982). Avant la pose des panneaux peints des grandes façades des maisons de culte, ils étaient associés à toutes sortes de feuillages colorés : croton, cordyline, crinum posés sur le sol devant l'édifice (Losche, 1982 : 59). Dans la région centre-nord Abelam, des fruits orange mban sont placés dans un panier sur la poutre faîtière d'une nouvelle maison de culte; ils représentent les noix d'arec de l'esprit nggwalndu (Hauser-Schäublin, 1986 : 2). Ces fruits verts et orange servent également lors des initiations et pour constituer de grandes figures, nggumaïra, réalisées directement sur le sol (Hauser-Schäublin, 1989a : 215, pl.VI ; 1989b : 146). Les fruits mban se retrouvent dans le rituel puti. Ils sont alors posés sur le sol au milieu d'anneaux de coquillages-monnaies ou fichés à la pointe de lances dont l'une est tenue par un mannequin anthropomorphe situé au centre de la maison de culte. Les Abelam, comme leurs voisins de l'ouest, les Yangoru, décorent leurs longues ignames masquées pour les présenter lors de grandioses cérémonies. Deux rangées de fruits mban, associés à de petites plumes blanches de poulet, sont fixées parallèlement à la longueur des tubercules.

Chez les Kwanga voisins, selon Schindlbeck (1985 : 384), lorsque la charpente d'une maison de culte est terminée, un homme grimpe à une échelle de l'échafaudage avant qu'elle soit recouverte de feuilles de palmier pour aller accrocher au sommet du faîtage un filet de fruits orange

2. Lors de leurs expéditions guerrières, les Asmat de Papouasie occidentale plaçaient à l'avant d'une pirogue un fruit orange non comestible. L'expédition se poursuivait tant que le fruit se maintenait. Si le fruit tombait à l'eau c'était un mauvais présage. 
d'Ervatamia . Lorsque la première moitié de la toiture est couverte, des hommes apportent un long bambou sur lequel sont accrochées des plumes de casoar, des fleurs d'hibiscus, des feuilles " décoratives » diverses et des feuilles de taro. D'autres hommes apportent une très longue javeline en bambou. Celle-ci est destinée à tuer les cochons qui seront sacrifiés ultérieurement. Ensuite, d'autres décorations sont encore apportées et accrochées sur le pignon de l'édifice, une longue guirlande de fruits orange et des feuillages d'ornement. Un paquet décoré de plumes blanches dans lequel se trouvent des substances magiques pour tuer les cochons est également suspendu à côté du filet. Après une danse circulaire, les hommes se placent en demi-cercle devant la nouvelle maison et le filet est vidé de ses fruits qui tombent alors que les hommes essayent de les repousser vers l'intérieur de l'édifice. Dans ce cas, les fruits représentent les cochons sauvages qui doivent être attrapés par les chasseurs. Ils sont donc utilisés à des fins propitiatoires. De même, dans la région de Kaugia, les fruits ban entrent dans les rites magiques pour la chasse au porc sauvage car ils sont en relation avec les esprits wale (Aufenanger, 1972 : 343-344). Dans la région de Kunjingini, les fruits ban de diverses couleurs sont considérés comme des objets de divination pour prévoir le nombre d'enfants que peut espérer une femme (Aufenanger, 1972 :414).

Les peuples vivant près du fleuve Sépik ont des représentations très similaires. Les Manambu utilisent lors des rituels Nimbi les fruits mbandj (Rejoua aurantiaca), empalés sur de courts bâtons pour représenter chacun un membre d'une généalogie (Harrison, 1982). Douglas Newton (1971: 82) confirme que les Nukuma perçoivent ces fruits analogiquement à des têtes humaines. Pour Ross Bowden (1983 : 159), les fruits bodii sont perçus de la même façon par les Kwoma qui les utilisent pour l'agencement des figures yena (Bowden 1983 : pl. 18) et les accrochent aux piliers centraux des nouvelles maisons (Bowden, 1984: 14). Ces fruits sont souvent représentés dans les peintures sur infrabases de palmier sagoutier ou gravés sur les poteries de certains groupes totémiques (Bowden, 2006 : 16-17, 21 et 76). Les Bahinemo les plaçaient sur les têtes sculptées de leurs grands tambours à fente lors des cérémonies d'initiation (Newton, 1971 : 20). Les Ngaala accrochaient ces fruits sous le porche de leurs maisons cérémonielles pour représenter les têtes humaines capturées durant les combats contre d'autres villages (Newton, $1971: 34$ ).
Nicolas Garnier évoque les fruits orange de Voaconga papuana dénommés bossikanunk (2007 : 300) qui sont utilisés chez les Chambri pour divers rituels. Ce fruit serait également appelé parfois yangenpandi, un mot formé avec le nom de la plante yangen (Ervatamia sp.) associé au suffixe pandi qui est employé pour désigner le rituel d'initiation. L'auteur regrette de ne pas avoir obtenu plus d'informations lui permettant de relier le terme pandi à la relation d'amitié pandi-pandi (Garnier, 2007 : 177) plus ou moins équivalente à la relation tambinien-tambinien des Iatmul voisins. Je pense que s'il avait observé plus attentivement un rameau de bossikanung, avec les fruits orange groupés par paires, il aurait compris immédiatement cette relation. En 1986, j'ai eu l'occasion d'observer l'utilisation de ces bossikanung lors d'une cérémonie destinée à accueillir l'évêque de Wewak à la mission de Chambri. Un des masques de proue de la pirogue double amenant le religieux était entouré d'une guirlande de fruits orange et le portique du débarcadère était surmonté d'une tête humaine en bois peint décorée de longues guirlandes de bossikanung orange, jaunes et verts.

\section{Conclusion}

Les représentations de ce concept de maturation et de murissement associé à la couleur d'un fruit ont vraisemblablement dépassé les frontières de la Papouasie Nouvelle-Guinée puisqu'elles semblent avoir existé jusqu'au Vanuatu et en Nouvelle-Calédonie. Dans sa description de la « décoration » du chemin menant à l'édifice du salagoro, lors des rites très importants et secrets des tamate aux îles Banks, Codrington (1972 : 77) évoque des fruits orange. Ces derniers, associés à des branches fleuries et à des frondes de cycas, étaient considérés comme la nourriture des morts (Vienne, 1984: 93). En Nouvelle-Calédonie, un fruit similaire était connu pour sa faculté à exprimer le processus vital d'assèchement inexorable de la naissance jusqu'à la mort. Le père Lambert évoque ce fruit comme une orange :

« Tous sont occupés à jouer avec des oranges : on ne connaît la différence des âges qu'à la couleur des fruits que l'on roule dans les doigts. Les premiers venus jouent avec des oranges sèches, ceux qui les ont suivis avec des oranges mûres et les derniers venus avec des oranges vertes. » (Lambert, 1976 : 14)

Dans le mythe de Pijeva, il est question d'un fruit similaire : 
«Ces dieux se lancent sans cesse une orange, d'espèce non comestible. Et celle-ci est verte, mûre ou desséchée, suivant qu'elle est lancée entre deux jeunes, adultes ou vieillards. » (Leenhardt, 1976 : 114)

Pourquoi les peuples du Sépik ont-ils focalisé leur attention sur ces genres de végétaux alors qu'ils disposaient dans leurs forêts de nombreuses autres espèces de plantes qui produisent des fruits dont la couleur vire du vert à l'orangérouge ? Peut-être avons-nous la réponse dans le fait que ces végétaux de la famille des Apocynacées ont en commun d'avoir un exsudat laiteux, des fleurs blanches et des fruits orangés, lisses et brillants, groupés par paires qui se détachent de leur support dès qu'ils ont atteint leur maturité, ensemble decaractéristiques quene possèdent pas d'autres végétaux à fruits orange. Si les ethnologues Bateson, Schindlbeck, Garnier et Juillerat évoquent des fruits de différents genres, il apparaît cependant que leurs données correspondent à un même champ de représentations. Il est probable qu'il existait, avant la christianisation des peuples du Sépik, une représentation plus ou moins générale de ces fruits orange proche de celle décrite par Bernard Juillerat chez les Yafar.

L'arbre Rejoua aurantiaca serait donc en relation avec le monde chthonien. Dans leur mythologie, les Yafar situent l'arbre Rejoua, issu du sang menstruel de la grand-mère originelle, comme planté à la bifurcation de deux chemins : l'un remonte vers la forêt au pays des esprits nabasa et l'autre conduit à l'entrée du monde des morts où les fantômes iffaf se trouvent être les gardiens des plantes cultivées durant la période de friche. C'est d'ailleurs grâce aux iffaf que les nouvelles pousses émergent de la bouture pourrissante (Juillerat, 1991: 200) et les données umeda recueillies par Gell s'accordent tout à fait avec cette vision yafar. De leur côté, les Iatmul et les Sawos enterraient sous les tertres et les monticules waak les corps de leurs ennemis tués. Leur pourrissement était censé apporter la prospérité à la communauté tout entière. Ces fruits de Rejoua étant associés aux pierres de fertilité chez les Abelam, il n'est pas étonnant qu'ils servaient à la divination pour prévoir les naissances des enfants dans la région de Kunjigini. Des mythes évoquent la pourriture de cadavres humains ou animaux comme terreau d'origine de nombreux végétaux (Coiffier, 1994: 14881490). Notons également que les Yafar comme les Iatmul accordent une grande importance à la dualité, donc au fait que tous ces fruits se présentent par paires sur leurs branches. Il n'est pas surprenant qu'ils associent l'arbre Rejoua à deux voies distinctes dans la déstructuration de la personne humaine lors de la mort. Il semble exister dans l'ensemble des peuples du Sépik une relation analogique entre les fruits orange caducs, le gibier et les ennemis tués.

Ces fruits ont une fonction proche de celle des "vanités ", images du XVII ${ }^{\mathrm{e}}$ siècle européen représentant parfois des crânes. Leur maturation rappelle aux humains qu'ils sont mortels et que leur corps est destiné à pourrir. Il est donc logique que ces fruits soient maintenant couramment utilisés dans les décorations de cérémonies religieuses chrétiennes. Mais, parfois, ces représentations se laïcisent puisque dans les écoles de brousse, lors des cours de travaux manuels, les instituteurs font maintenant réaliser à leurs élèves des objets divers à l'aide de fruits ronds de Rejoua assemblés avec des baguettes. Bernard Juillerat eut l'opportunité d'étudier la société yafar avant sa christianisation. Nous avons la chance qu'il ait pu nous transmettre les conceptions eschatologiques d'un peuple dont la pensée n'avait pas encore été influencée par l'idéologie chrétienne.

\section{Remerciements}

Je remercie tout particulièrement Lucille Allorge, attachée au Muséum national d'histoire naturelle, pour les informations qu'elle m'a aimablement transmises au sujet de la famille des Apocynaceae à laquelle appartiennent la majorité des végétaux cités dans ce texte. 
Annexe : Noms d'arbres à fruits orange de la famille des Apocynaceae dans quelques langues de la région du Sépik

\begin{tabular}{|l|l|l|}
\hline Nom d'ethnie ou de village & Nom local du Rejoua sp. & Groupe linguistique \\
\hline Arapesh & su'witip & Arapesh \\
\hline Abelam & mban ou ban & Ndu \\
\hline Biwat & mawut & Yuat \\
\hline Dimiri & opabu & Mongol-langam \\
\hline Chambri & bossikanung & Pondo \\
\hline Iatmul & mbwandi & Ndu \\
\hline Kapriman & bolmeur & Sepik-hill \\
\hline Kwoma & bodi & Nukuma \\
\hline Manambu & mbandj & Ndu \\
\hline Ngaala & mbaal & Ndu \\
\hline Umeda & subove & Waris \\
\hline Wogamush & mbigli & Wogamusin \\
\hline Yafar & boof & Amanab \\
\hline Yangoru & ban & Ndu \\
\hline
\end{tabular}

Le phonème « $\mathrm{b}$ » est présent dans la majorité des noms désignant le Rejoua aurantiaca (Coiffier, 1996 : 118, 120).

\section{BIBLIOGRAPHIE}

Allorge Lucille, 1985. Apocynaceae-Tabernaemontanoideae. Origine de l'arille et ornementation du tégument séminal, Bulletin du Museum national d'histoire naturelle de Paris, $4^{\mathrm{e}}$ série (7), pp. 434-451.

Aufenanger Henry, 1972. The Passing Scene in North-East New-Guinea (A Documentation), St. Augustin, Anthropos Institute, Instituti Anthropos 2.

BATESON Gregory, 1958 ( $1^{\text {st }}$ published in 1936). Naven. A survey of the problems suggested by a composite picture of the culture of a New Guinea tribe drawn from three points of view, Stanford (California), Stanford University Press (trad. française de J.-P. Latouche et N. Safouan, 1971, La cérémonie du naven, Paris, éditions de Minuit).

Bowden Ross, 1983. Yena. Art and Ceremony in a Sepik Society, Oxford, University of Oxford, Pitt Rivers Museum, Monogr. 3.

—, 2006. Creative Spirits. Bark Painting in the Washkuk Hills of North New Guinea, Melbourne (Australia), Océanic Art Pty Ltd.

Codrington R.H., 1972 ( $1^{\text {st }}$ published in 1889). The Melanesians, Studies in their Anthropology and folklore, New-York, Dover Publications Inc.

CoIfFIER Christian, 1994. L'écorce et la moëlle du rotin (tshimbe kuvu, kwiya kuvu), conception iatmul de l'univers (Papouasie), doctorat en anthropologie sociale et en ethnologie, Paris, EHESS.

—, 1996. Vegetal Names in the Ndu Languages, MonKhmer Studies. A Journal of Southeast Asian Languages XXv: Special Volume Dedicated to Professor André-Georges Haudricourt, pp. 109-124.
COOPER Wendy and William T. COOPER, 2004. Fruits of the Australian Tropical Rainforest, Melbourne, Victoria, Australia, Nokomis Editions.

Forge Anthony, 1971. Art and Environment in the Sepik, in Carol F. Jopling (eds), Art and Aesthetics in Primitive Societies. A critical anthology, New-York, E.P.Dutton, pp. 291-314.

GARDI René, 1958. Sepik. Land der Sterbenden Geister, Zürich, Büchergilde Gutenberg.

GARNIER Nicolas, 2007. Étude des concepts soustendant la vie sociale des Chambri (East Sepik Province, Papouasie Nouvelle-Guinée), Paris, thèse de doctorat du Muséum national d'histoire naturelle.

Gell Alfred, 1975. Metamorphosis of the Cassowaries. Umeda Society, Language and Ritual, London, Athlone Press/ New Jersey, Humanity Press, London School of Economics, Monographs on social Anthropology 51.

Guise G. B., M. Rasmussen, F. Richie and W.C. TAYLOR, 1965. Some constituants of Rejoua aurantiaca and Voacanga papua, Australian Journal of Chemistry 18 (6), pp. 927-931.

Harrison Simon J., 1982. Stealing People's Names: Social Structure, Cosmology and Politics in a Sepik river Village, thesis submitted for the Degree of Doctor of Philosophy of the Australian National University, multigr.

HAUSER-SCHäUBLIN Brigitta, 1986. The visual expression of man's relation to death and the world of spirits, paper for the symposium 101: Sepik Culture History: Variation and Synthesis, 15-24 fév. 1986, Mijas (Espagne), W.G.F.

-, 1989a. Kulthäuser in Nordneuguinea, Berlin, Akademie-Verlag. 
—, 1989b. Leben in Linie Muster und Farbe. Einführung in die Betrachtung aussereuropaïscher Kunst, am Beispiel der Abelam Papua-Neuguinea, Basel/Boston/Berlin, Birkhäuser Verlag.

Hauser-SchäUblin Brigitta et Jörg HauserSchäUBlin, 1980. Wir und unser Dorf. Die Kinder der Abelam in Papua Neuguinea, Basel, Lehrmittelverlag des Kantons Basel-Stadt.

JuILlERAT Bernard, 1982. Note sur les rapports de production dans l'horticulture-arboriculture yafar (Nouvelle-Guinée), Journal d'agriculture traditionnelle et de botanique appliquée 30, 1, pp. 285-293.

-, 1983. L'essartage chez les Yafar (NouvelleGuinée), Journal d'agriculture traditionnelle et de botanique appliquée XXX (1), pp. 4-33.

—, 1984a. Culture et exploitation du palmier sagoutier dans les Border Mountains (Nouvelle-Guinée), Techniques \& culture 3, pp. 43-64.

_, 1984b. D'Acorus à Zingiber. Taxinomie et usages des plantes cultivées chez les Yafar de NouvelleGuinée, Journal d'agriculture traditionnelle et de botanique appliquée XXXI (1-2), pp. 3-31.

_, 1986. Les enfants du sang. Société, reproduction et imaginaire en Nouvelle-Guinée, Paris, Editions de la maison des sciences de l'homme.

—, 1991. Edipe chasseur. Une mythologie du sujet en Nouvelle-Guinée, Paris, PUF.

_, 1992. Shooting the Sun. Ritual and Meaning in West-Sepik, Washington/London/Paris, Smithsonian Institution Press in cooperation with the ministère des Affaires étrangères.

_, 1995. L'avènement du père. Rite, représentation, fantasme dans un culte mélanésien, Paris, CNRS Éditions/ Éditions de la maison des sciences de l'homme.
KIRK Malcolm S., 1973. Change Ripples New Guinea's Sepik River, National Geographic Magazine 144 (3), pp. 354-381.

LAMBert S. M., 1976 (1 $1^{\text {ère }}$ éd. 1900). Mours et superstitions des Néo-Calédoniens, Nouméa, Société d'études historiques de la Nouvelle-Calédonie.

LeEnHARdt Maurice, 1976 (1 $1^{\text {ère }}$ éd. 1947). Do Kamo. La personne et le mythe dans le monde mélanésien, Paris, Gallimard.

Losche Diane, 1982. The Abelam, a people of Papua New Guinea, Sydney, A. Bishop Ed., The Australian Museum.

Newton Douglas, 1971. Crocodile and Cassowary, Religious art of the Upper Sepik River, New Guinea, New York, The Museum of Primitive Art.

Paijmans K., 1976. New-Guinea Vegetation, Canberra, National University Press.

SCHINDLBECK Markus, 1985. Männerhaus und weibliche Giebelfigur am Mittelsepik, Papua-Neuguinea, Ethnologica, Neue Folge, Band XXXIII, pp. 363411.

SCHMID Jürg et Christin KocHER-SCHMID, 1992. Söhne des Krokodils. Männerhausrituale und Initiation in Yensan, Zentral-Iatmul, East Sepik Province, Papua New Guinea, Basel, Basler Beiträge zur Ethnologie, Band 36.

Van Royen P., 1969. Manual of the Forest Trees of Papua and New Guinea, Part. 9, Apocynaceae, Lae/Port Moresby, Deparment of Forests, Administration of Papua and New Guinea.

VIENNE Bernard, 1984. Gens de Motlav. Idéologie et pratique sociale en Mélanésie, Paris, Société des Océanistes, Publication de la Société des Océanistes 42. 
